\title{
Changes over time in thresholds for electrical stimulation of the cochlea
}

\author{
Bryan E. Pfingst \\ Kresge Hearing Research Institute, Department of Otolaryngology, University of Michigan Medical Center, Ann Arbor, Michigan, U.S.A.
}

(Received 28 February 1990; accepted 22 June 1990)

\begin{abstract}
The purpose of this paper is to better characterize changes over time that occurred in psychophysical detection thresholds for electrical stimulation of the cochlea. Threshold changes observed in nonhuman primates implanted with cochlear electrode arrays can be divided into at least three types based on the patterns of change over time. Short-term increases and subsequent decreases in threshold were commonly observed during the first months after implantation and were often followed by periods of long-term threshold stability. Long-term slow increases in thresholds and more rapid increases after a period of threshold stability have also been observed. The threshold changes may be divided into at least two classes based on their dependence on the waveforms used for the threshold measurements. Some changes occurred primarily in thresholds for long phase-duration signals while other changes were equal in magnitude (in decibels) for all tested stimuli. This suggests that at least two mechanisms underlay these threshold changes. The observed changes in thresholds have implications for experimental studies of electrical stimulation and for clinical application of auditory prostheses.
\end{abstract}

Auditory prosthesis; Electrical stimulation; Nonhuman primate; Psychophysics; Threshold

\section{Introduction}

Psychophysical detection thresholds for eletrical stimulation of the nervous system, as observed in subjects with electrode arrays implanted in or near the cochlea, are usually characterized by their long-term stability. There are, however, some clear exceptions. We have reported previously on relatively rapid systematic fluctuations in thresholds that occur in the first few weeks or months following deafening of the cochlea, implantation of electrodes, and electrical stimulation, in nonhuman primates (Pfingst et al., 1979). There are also reports of decreases in thresholds shortly after implantation in human subjects (Eddington et al., 1978; Michelson, 1971). In addition to these short-term changes, we have seen long-term changes in thresholds in a smaller number of

Correspondence to: Bryan E. Pfingst, Kresge Hearing Research Institute, 1301 East Ann Street, Box 0506, University of Michigan, Medical Center, Ann Arbor, MI 48109-0506, U.S.A. cases. These fall into two categories: slow increases in thresholds over periods of months or years; and more rapid increases in thresholds occurring over periods of a few days or weeks preceded and/or followed by periods of threshold stability. These latter two types of changes have not been reported previously in any detail.

Such changes in thresholds have implications for the design and calibration of auditory prosthesis processors, for monitoring the condition of the implanted cochlea, and for experimental studies of cochlear electrical stimulation. They may prove useful in studying the neural mechanisms underlying stimulus detection. To better understand the nature and implications of these changes we have sought to describe their characteristics in more detail. In particular, in this paper we will focus on their dependence on the stimulus waveforms used to measure thresholds. The mechanisms underlying the changes are unknown, though some hypotheses can be formed based on their characteristics.

A report of some of these findings was given previously (Pfingst, 1989a). 


\section{Methods}

\section{Overview}

The data reported in this paper are based on observations made over a 12-year period of study involving 36 implants in 28 nonhuman primates (macaques). Thresholds for all of these implants were followed for periods ranging from 3 months to several years following implantation. The exact protocols differed from subject to subject depending on the experimental design, which often involved other psychophysical measures in addition to threshold. The protocols ended prematurely in some cases due to implant failures, but all of the cases reported here were studied for at least 3 months. General descriptions will be based on the whole population while more detailed observations will be reported for a few of these subjects.

The subjects were trained psychophysically using positive reinforcement operant conditioning procedures to perform psychophysical tasks that could be used to measure thresholds, dynamic ranges, and various difference limens (Pfingst et al.. 1979, 1983; Pfingst, 1989b). The subjects were trained using acoustic stimuli and then they were deafened in one ear and implanted with a multielectrode array in the scala tympani and/or in the bony cochlear wall. In some cases, the contralateral ear was implanted several months or years following implantation of the first ear. In the other cases one ear was left untreated and was used for additional training and testing with acoustic stimuli. In a few cases, the implant failed some months following the initial surgery and the ear was subsequently reimplanted with another electrode array. Typically, thresholds for electrical stimuli were measured as a function of time following implantation until stable, and then a variety of psychophysical data, including thresholds, were collected over a period of several years. Electrode impedances were measured during each experimental session at the frequencies for which psychophysical testing was conducted during that session and at a fixed low current level.

\section{Subjects}

The subjects were adolescent or adult macaques ( $M$. mulatta and $M$. radiata). They were housed in individual primate cages except during training and testing sessions at which time they were seated in primate chairs.

\section{Apparatus}

During training and testing sessions the subjects sat in double-walled sound attenuating chambers (IAC type 1201A or Tracoustics model RE-240-B). A light-display panel was positioned in front of the subjects, a telegraph key was located within reach of either hand, and a tube for delivery of applesauce reinforcers was positioned near the mouth. For acoustic stimulation, ear speakers (Beyer Dynamic DT 48 or TDH 49) fitted with circumaural cushions (Pfingst et al., 1975) were positioned against the subject's head. Sinusoidal stimuli were generated by a Rockland frequency synthesizer and gated by a tone switch built in house. Rectangular pulses were generated by computer or by electronics built in house. These signals were attenuated by a computer controlled attenuator (Grason-Stadler Model 1284 or Wilsonics model PATT), and then passed to the earspeaker or to a constant current stimulator (similar to the design described by Spelman et al., 1978). For electrical stimulation, the output of the constant current stimulator was connected directly to the implants through a percutaneous connector.

Experimental paradigms and data collection were computer controlled.

\section{Psychophysical procedures}

Thresholds were measured using a go, no-go procedure in which the subject depressed a telegraph key to initiate each trial, held the key during a randomly variable (1-6 s) foreperiod (observing response), and released the key at stimulus onset signaling detection of the stimulus. The stimulus remained on until the subject responded, or a maximum of $1 \mathrm{~s}$, except in cases where the paradigm required a shorter stimulus (e.g. single pulses). Responses (releases within $1 \mathrm{~s}$ of stimulus onset) were reinforced by delivery of $0.2 \mathrm{cc}$ of applesauce to a spout located near the subject's mouth. Percent responses were plotted as a function of stimulus level to form psychometric func- 
tions and threshold was defined as the level on the psychometric function at which the subject responded on $50 \%$ of the trials. Stimuli were presented by the method of constant stimuli. Stimulus tables were constructed so as to maintain a relatively constant rate of reinforcement across conditions in order to avoid conditions which might lead to a change in response strategy. Twenty trials per stimulus were used to construct the psychometric functions. Guess rates (releases during the $1 \mathrm{~s}$ unmarked observation period on trials where no stimulus was presented) were measured during all sessions. We attempted to keep the guess rate constant by controlling the duration of a penalty time out, contingent on early releases. With this method, guess rates were usually kept within a range of $2 \%$ to $15 \%$ and did not vary systematically across conditions. If these criteria for the guess rate were not met, the data were not used and additional training was carried out.

\section{Implants and surgical procedures}

Surgery for deafening and implanting the ears was performed under halothane anesthesia using standard sterile operating procedures. After the subject was prepared and draped, a post-auricular incision was made. In early surgeries mastoidectomy was carried out to reach the middle ear while in later surgeries the bony external meatus was merely enlarged. In the latter cases, the peripheral portion of the meatus soft tissue was sutured closed. Soft tissue was then removed from the medial part of the meatus and the bony meatus was enlarged to provide a wide exposure of the middle ear. The incus was carefully disarticulated from the stapes and the malleus and incus were removed leaving the stapes in the oval window. In most cases, $50 \mu 1$ of a $10 \%(w / v)$ neomycin sulfate solution was slowly injected into the scala tympani via a 30 -gage needle which penetrated the round window. In four cases, no neomycin was administered. For scalar implants, the round window was removed and the electrode array was inserted. In some cases electrodes were implanted in holes drilled in the bony wall of the cochlea. Multichannel scalar implants consisted of platinum-iridium electrodes on a silicone-rubber carrier about 10 $\mathrm{mm}$ in length and approximately half the diameter of the scala tympani (Xue and Pfingst, 1989). In a few early cases the silicone carrier was molded to a monkey cochlea following the design described by Michelson (1971). The scalar implants were inserted through the round window, 5-10 $\mathrm{mm}$ past the round window niche. Electrodes inserted in the cochlear wall or single electrodes inserted though the round window consisted of spherical platinum-iridium electrodes approximately $0.3 \mathrm{~mm}$ in diameter. Wires from all electrodes were led under the skin to a percutaneous connector mounted on the skull (Pfingst et al., 1989).

\section{Results}

\section{Types of change}

Changes over time observed in our experiments were classified into three types based on the timecourse of the change. One type, which we will call short-term changes, occurred during the first few months following implantation of the electrode array, starting as soon as testing began. Examples of this type of change have been published previously (see Fig. 3 in Pfingst et al., 1979). In cases where threshold measurements were begun within the first week after the implant surgery, we usually observed an initial rise in thresholds over a period of several days (see Incidence section for details). Within 1 to 5 weeks after surgery the thresholds began to decrease at rates on the order of $0.2-1.0$ $\mathrm{dB} /$ day. This period of decreasing thresholds lasted for one to several months, after which thresholds typically stabilized. This short-term decrease in thresholds (often preceded by an initial increase) was seen in most of the cases we studied. It was seen after initial implants (e.g. Fig. 1) and after reimplantation (e.g. Fig. 2). Acoustic thresholds measured in the subjects' nonimplanted ears during the period when electrical thresholds were changing were typically stable. Implant impedances measured during the first months following implantation were typically not correlated, or only weakly correlated with the threshold changes.

The other two categories of changes occurred more rarely and followed the short-term changes. One of these was a prolonged increase in thresholds that occurred with some regularity over a period of several years. An example of this type 


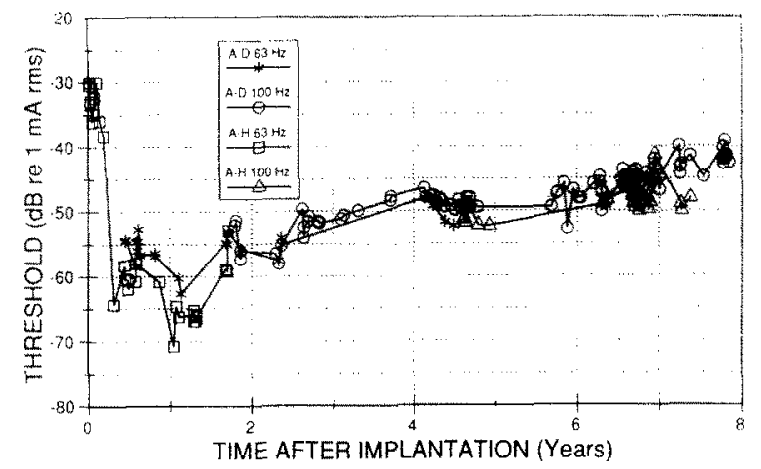

Fig. 1. Thresholds plotted as a function of time after implantation for implant AB-L-1. The first thresholds were measured 4 days after the surgery in which the ear was neomycin deafened and implanted. The electrode array consisted of one electrode (electrode D) in the scala tympani just inside the round window, one (electrode $B$ ) in a hole in the bone near the second turn of the cochlea, one (electrode A) in a hole in the bone near the apex, and a remote electrode (electrode $H$ ) in the parietal bone. Thresholds for two electrode pairs (A-D and A-H) at two similar frequencies of simusoidal stimulation $(63$ and $100 \mathrm{~Hz}$ ) are shown.

of change is shown in Fig. 1. In this case, after the initial drop in thresholds and some fluctuations during the first year and a half after implantation,

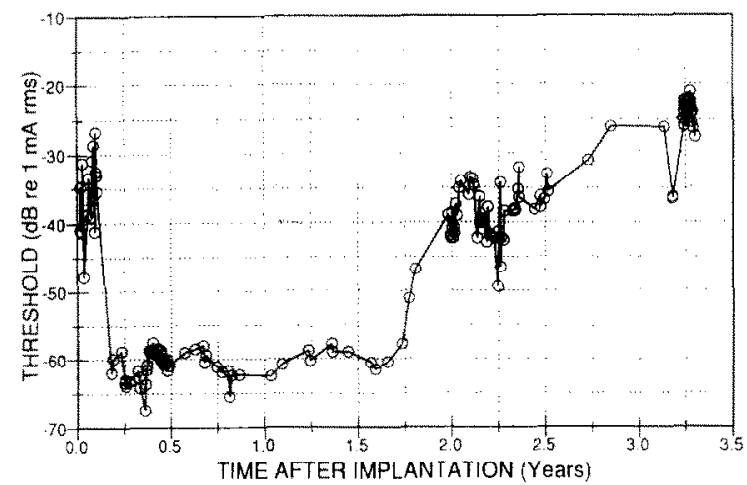

Fig. 2. Thresholds plotted as a function of time after implantation for implant QU-R-3. The first thresholds were measured 6 days after a surgery in which the ear was implanted with a scala tympani electrode array. This ear had been neomycin deafened and implanted with a different scala tympani electrode array 8 months previously, but that implant was removed following a failure of the percutaneous connector. The cochlea also contained 3 titanium cochlear-wall electrode holders (Pfingst et al., 1989b) which were sealed and not used for stimulation. All thresholds are for $100 \mathrm{~Hz}$ sinusoidal bipolar electrical stimulation between two electrodes located $1.5 \mathrm{~mm}$ apart and placed in the basal turn of the scala tympani. thresholds for low-frequency stimuli began a slow steady rise. Over the period from 1.8 ycars to 7.8 years after implantation, thresholds for $100 \mathrm{~Hz}$ sinusoids for electrode pair A-D rose about $12 \mathrm{~dB}$. A least squares regression line fit to the data points during this period had a slope of 1.96 $\mathrm{dB} /$ year. Acoustic thresholds for $1 \mathrm{kHz}$ pure tones to the nonimplanted ear showed no change during this period. Impedances for this implant rose during the first 3 weeks after implantation and then stabilized, remaining relatively stable throughout the period when the psychophysical thresholds decreased and then showed a gradual rise. The correlation between psychophysical thresholds and impedances measured during the period when thresholds were rising gradually was 0.32 .

A third category of change consisted of a more rapid rise in thresholds that occurred over a period of several days or weeks and that sometimes followed a long period of threshold stability. An example of this type of rise is shown in Fig. 2. In this example, following some initial fluctuations in the first month after implantation, thresholds dropped to a level of about $1 \mu \mathrm{A} \mathrm{rms}$ and remained relatively stable for a period of about 1.5 years. Thresholds then rose about $20 \mathrm{~dB}$ over a period of about 4 months. After that, thresholds were less stable and, on average, continued to rise until the implant was removed due to a mechanical failure of the percutaneous connector. Acoustic thresholds for a $1 \mathrm{kHz}$ pure tone to the nonimplanted ear remained stable during this period. Impedance of the implant showed no systematic change during the period when the psychophysical thresholds were rising.

\section{Incidence}

In 34 cases, thresholds for at least one frequency $(63 \mathrm{~Hz}, 100 \mathrm{~Hz}$ or $1 \mathrm{kHz}$ ) of sinusoidal stimulation were followed over a period starting during the first week after implantation of the electrodes and extending for 3 months or more. In $30(88 \%)$ of these cases, thresholds were highest during a period sometime during the first month after implantation and then decreased by 8 to $37 \mathrm{~dB}$ during the following weeks. In 4 of the 34 cases observed during this initial period, thresholds showed only small changes ( $<8 \mathrm{~dB}$ ), or no systematic changes 
from the first day of testing ( 1 to 4 days postsurgery) and remained stable for as long as testing continued.

In 18 of the 34 cases threshold testing began within 1 to 4 days following surgery. In 14 of these cases $(78 \%)$ we saw initial increases in threshold at rates of 0.5 to $6.0 \mathrm{~dB}$ per day peaking at an average of 16 days post-surgery. The remaining 4 cases showed no systematic changes in threshold as mentioned above. In 16 cases where threshold measurements were not begun until 5 to 8 days after surgery, thresholds were initially high and then decreased over time or fluctuated for a period of time and then decreased.

In two cases, threshold testing was not begun until about 2 months after the implant surgery, and in those cases thresholds for $1 \mathrm{kHz}$ sinusoids were stable (showed no systematic increases or decreases) from the first day of testing.

Twenty cases were followed for periods of 100 days or more after the threshold decrease (or after testing began, in cases where thresholds were initially stable). In 15 (75\%) of these cases, after the thresholds reached a minimum they remained stable for as long as testing continued (100-800 days). In three (15\%) of the cases we saw a slow, prolonged increase in thresholds following the initial decrease (as in the example in Fig. 1). In two other cases $(10 \%)$ we saw increases in thresholds

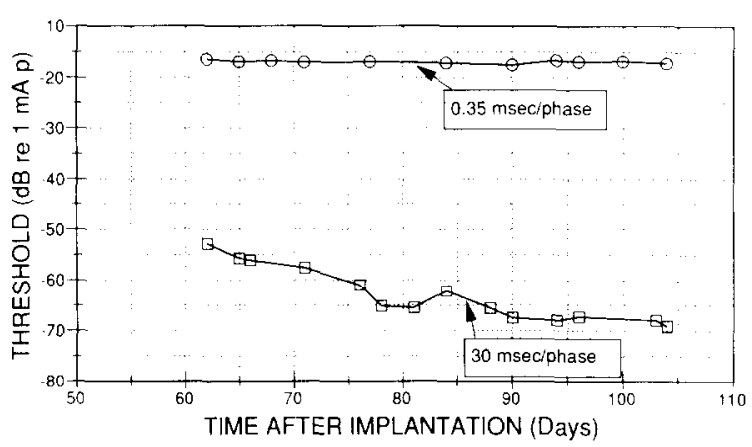

following periods of threshold stability (as in the example in Fig. 2).

\section{Effects of waveform}

\section{Short-term changes}

In most cases we followed thresholds as a function of time at only one frequency of sinusoidal stimulation $(63 \mathrm{~Hz}, 100 \mathrm{~Hz}$ or $1 \mathrm{kHz})$ until the thresholds were stable. However, in three cases we began measuring thresholds for other waveforms before threshold stability was achieved. In these cases we found that the amount of change differed for different waveforms. Specifically, thresholds for low frequency sinusoids or long phase-duration pulses changed more as a function of time. and/or changed over a longer period of time, than those for short phase-duration signals. An example is shown in Fig. 3. In this case, thresholds for single biphasic pulses (one pulse per trial) were measured as a function of phase duration over a 2-month period starting about 2 months after implantation. At the time these measurements were begun, thresholds for $1 \mathrm{kHz}$ sinusoids had reached a stable level. During the period when thresholds for the pulses were collected, thresholds for the longest duration pulses decreased by about $16 \mathrm{~dB}$ while those for short duration pulses showed no systematic change.

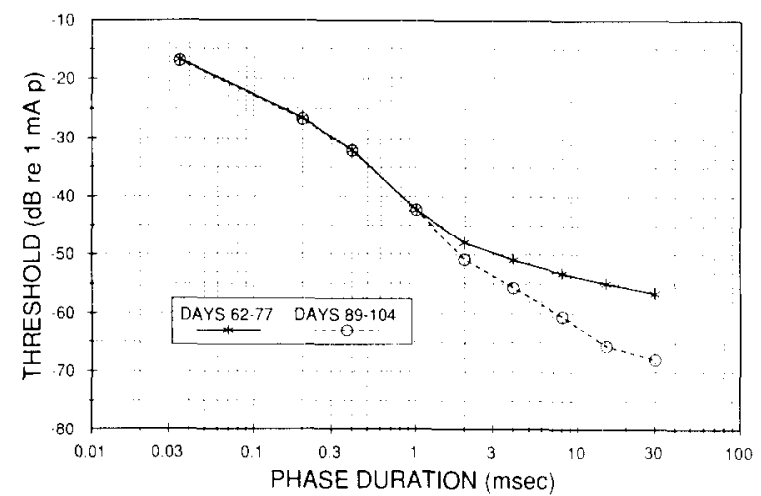

Fig. 3(A) Thresholds plotted as a function of time after implantation for implant PO-R-1, electrode E. monopolar stimulation. The thresholds are for single symmetrical biphasic pulses (one pulse per trial). The electrode was located in the scala tympani approximately $5 \mathrm{~mm}$ from the round window. After this ear was deafened with neomycin and implanted, thresholds for $1 \mathrm{kHz}$ sinusoids were followed over time until stable before the data for pulses (shown here) were collected. (B) Threshold contours (psychophysical detection threshold vs. phase duration) for single pulses (one pulse per trial) for implant PO-R-1, electrode E, monopolar stimulation, measured at two time periods following deafening and implantation of the ear. The periods during which the contours were collected are indicated in the legend. Mean thresholds for five measurements at each phase duration during each period are shown. Thresholds for two of the pulses are plotted as a function of time in (A). 
Further evidence that short-term changes in threshold depend on the waveform used to measure threshold comes from comparisons of changes over time for groups of subjects tested at $63 \mathrm{~Hz}$ or $100 \mathrm{~Hz}$ vs. those tested at $1 \mathrm{kHz}$. The average drop in thresholds from the initial peak to the final stable level was $20.7 \mathrm{~dB}$ (range $=8$ to $37 \mathrm{~dB}$ ) for cases measured at 63 or $100 \mathrm{~Hz}$ while that for thresholds measured at $1 \mathrm{kHz}$ averaged $13.6 \mathrm{~dB}$ (range $=8$ to $21 \mathrm{~dB}$ ). In addition, we found that the period over which thresholds dropped was longer on average for thresholds measured at 63 or $100 \mathrm{~Hz}$ (mean time $=63$ days, range $=6$ to 160 days) than for those measured at $1 \mathrm{kHz}$ (mean time $=25$ days, range $=5$ to 37 days).

\section{Long-term changes}

Long-term changes in threshold fell into two categories with regard to their dependence on waveform: those for which the changes were greatest for, or occurred only for, low frequency sinusoids (e.g. Fig. 4), and those for which the threshold changes were roughly the same magnitude (in $\mathrm{dB}$ ) for all frequencies tested (e.g. Fig. 5).

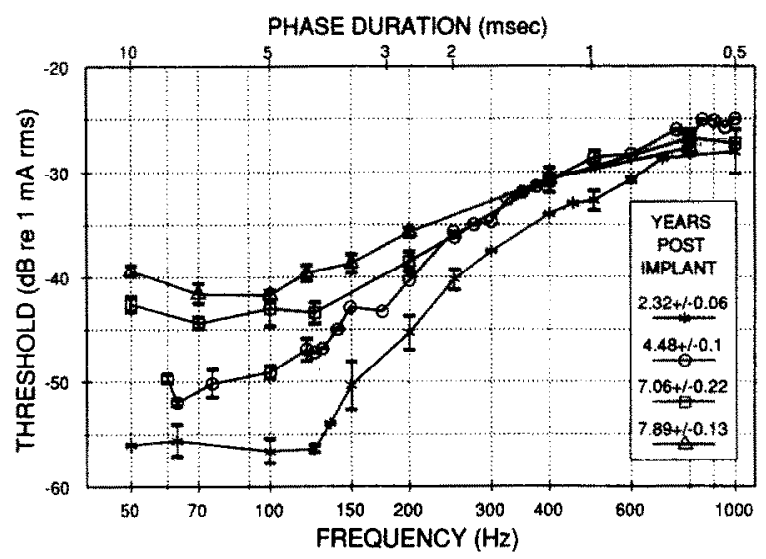

Fig. 4. Threshold contours (threshold vs. frequency and phase duration) for sinusoidal electrical stimulation of implant AB-L1, electrode pair A-D collected at four different time periods following implantation. Mean thresholds \pm 1 S.D. are shown, The time periods during which the contours were collected are indicated in the legend which gives the median of the time period, relative to the implant surgery, over which the data were collected, plus and minus half of the range of that time period. The threshold vs. time contours for low-frequency signals for this implant are shown in Fig. 1.

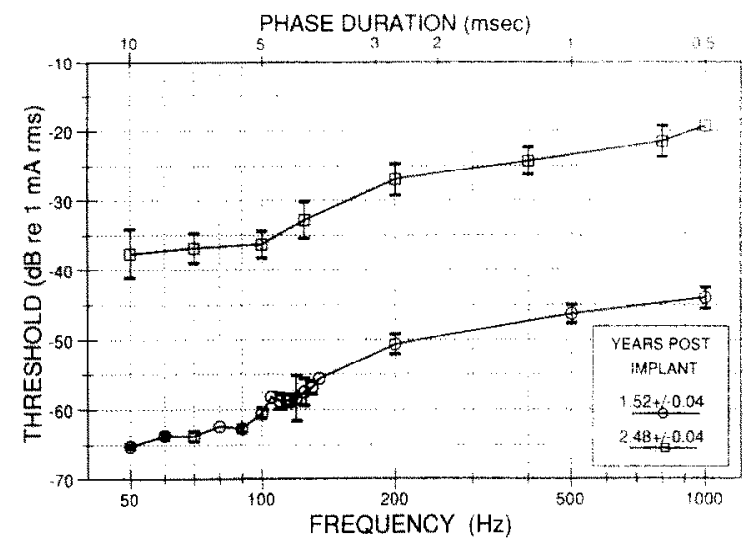

Fig. 5 Threshold contours (threshold vs. frequency and phase duration) for sinusoidal electrical stimulation of implant QUR-3, electrode pair A-C. Mean thresholds \pm 1 S.D. are shown. The periods during which the contours were collected are indicated in the legend as in Fig. 4. The threshold vs. time contour for $100 \mathrm{~Hz}$ sinusoids for this implant is shown in Fig. 2.

The case represented in Figs. 1 and 4 and some of the following figures (implant AB-L-1) has been studied in more detail than any other with regard to the dependence of the threshold changes on waveform. This detailed study was facilitated by the fact that the thresholds for this subject changed very slowly over time (about $2 \mathrm{~dB} /$ year, Fig. 1) so that a reasonably large data set could be collected over a period of a month or more while average thresholds changed by less than $1 \mathrm{~dB}$. These measurements could then be repeated at a later time (e.g. several years) after thresholds for some stimuli had increased.

Changes over time in the contours for sinusoids for implant AB-L-1 are shown in Fig. 4. Over a period of 5.5 years thresholds for frequencies below $100 \mathrm{~Hz}$ increased by about $15 \mathrm{~dB}$ while those at frequencies above $500 \mathrm{~Hz}$ increased by less than $3 \mathrm{~dB}$.

In contrast, thresholds for implant QU-R-3 (Figs. 2 and 5) increased, following a period of threshold stability, by about the same amount $(26$ to $28 \mathrm{~dB}$ ) at all frequencies tested. This type of uniform change (in $\mathrm{dB}$ ) across frequency was seen in one other case where thresholds rose after a long period of stability.

Further comparisons over time for implant ABL-1, where the threshold changes were frequency- 
dependent, revealed more about the properties of the signals for which the threshold changes occurred. Changes over time in the contours for the single biphasic symmetric pulses are shown in Fig. 6. In these tests the subject was presented with only one biphasic pulse per trial and thresholds were measured as a function of phase duration. The largest changes were for the longest duration pulses tested, the $10 \mathrm{~ms} /$ phase pulses. The change in mean thresholds for this pulse over this 2.6 year time period was $3.7 \mathrm{~dB}$. For shorter duration pulses, the changes were smaller or nonexistent. An analysis of variance with contrasts showed significant differences $(P<0.05)$ between the two time periods for the 10,5 , and $3 \mathrm{~ms}$ phase durations but not for the 2 and $1 \mathrm{~ms}$ phase durations. Note that the change in thresholds for the 10 $\mathrm{ms}$ /phase pulses over the 2.6-year period was smaller than the $6 \mathrm{~dB}$ change in threshold for the lowest frequency sinusoids observed over this same time period (Fig. 4).

Changes over time in the threshold contours (threshold vs frequency) for fixed-duration biphasic pulses ( $2 \mathrm{~ms} /$ phase) are shown in Fig. 7. These contours have the nonmonotonic shape typical of threshold contours for long duration pulses (e.g. see Shannon, 1989). For these con-

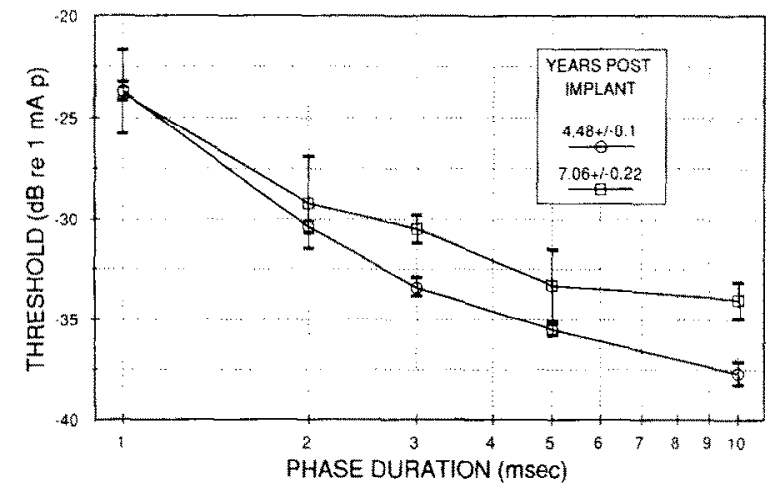

Fig. 6. Threshold contours (threshold vs. phase duration) for single symmetrical biphasic electrical pulses to implant AB-L-1, electrode pair A-D. Mean thresholds \pm 1 S.D. are shown. The time periods during which the contours were collected are indicated in the legend as in Fig. 4.

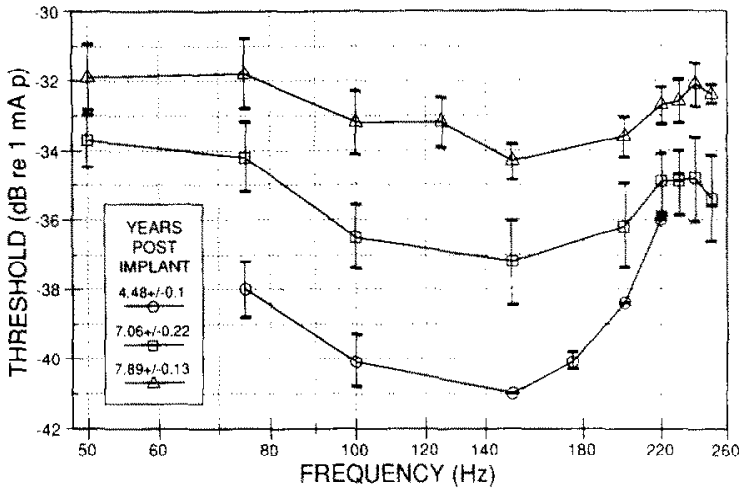

Fig. 7. Threshold contours (threshold vs. frequency) for trains of fixed-duration ( $2 \mathrm{~ms} /$ phase) electrical pulses for implant AB-L-1, electrode pair A-D. Mean thresholds \pm 1 S.D. are shown. The time periods during which the contours were collected are indicated in the legend as in Fig. 4.

tours, a relatively constant change in threshold occurred at frequencies from 100 to $150 \mathrm{~Hz}$ but above and below these frequencies, the changes were smaller. The magnitude of the change in thresholds for the fixed duration pulses over the period from 4.48 years to 7.8 years post-implant was about $7 \mathrm{~dB}$. This is roughly equivalent to the change seen in low frequency $(100-150 \mathrm{~Hz})$ sinusoids over approximately this same period, but much larger than the changes in thresholds for $250 \mathrm{~Hz}$ sinusoids, which have a phase duration of $2 \mathrm{~ms}$.

\section{Discussion}

\section{Patterns and incidence}

It is very clear from the data presented here that changes in threshold over time can take a variety of forms both in the pattern of change as a function of time and in the dependence of the changes on the waveforms for which the thresholds are measured. One implication of this variety is that incidence can be clearly specified only if the criteria for threshold stability are given. On the basis of our experience with nonhuman primates, we can suggest some guidelines that may be useful in testing for threshold stability: 
Long phase-duration signals (sinusoids near $100 \mathrm{~Hz}$, or pulses on the order of $5 \mathrm{~ms} /$ phase) * should be included in tests for threshold stability since they are sensitive to most, if not all, threshold changes and are the only stimuli that will permit detection of some types of changes.

- Within-subject variance in threshold measures should be assessed to establish confidence intervals for threshold change for each subject and then thresholds should be monitored over sufficient time periods to allow slowly changing thresholds to exceed those confidence limits. Since rates of threshold change can be at least as low as 1-2 $\mathrm{dB}$ per year, several years of monitoring may be required to detect such changes.

Since these criteria have been applied in few cases, it is difficult to estimate the incidence of threshold changes in most populations of implanted subjects.

Our observations of threshold changes in nonhuman primates do not necessarily mean that threshold changes over time occur in other populations such as other research animal preparations or human subjects. Certainly, there were some important differences between our animal preparation and some other implanted subjects including the use of local perfusion of neomycin to deafen the ears in our subjects at the time of implantation (although this was not done in all cases) and the fact that our subjects were stimulated electrically for only a fraction of a $2-4 \mathrm{~h}$ period each day. Possible implications of these procedures will be discussed below.

It would be premature at this time to say whether there is a relationship between the temporal patterns of threshold change and the dependence of the changes on stimulus waveform because detailed data on the effects of waveform were limited to only a few subjects.

All of the thresholds reported in this paper are in $\mathrm{dB}$ of current. Decibels or other logarithmic scales are commonly used for plotting thresholds

\footnotetext{
* Safety and certain other practical considerations place upper limits on the phase durations which can be used. However, signals with phase durations on the order of $5 \mathrm{~ms}$, which correspond approximately to the fundamental frequency of the voice for male speakers, are commonly used in some analog processors.
}

for electrical stimulation of the cochlea. The shapes of the threshold contours would, of course. be different if another scale were used. In particular, note that changes in threshold which are even for all frequencies on a dB scale (e.g. Fig. 5) are larger in linear microamps at high frequencies. However. in most cases where changes over time were greater in $\mathrm{dB}$ at low frequencies these changes would also be greater in linear current at low frequencies since often in these cases no change. or even a slight decrease in threshold over time, was seen at high frequencies.

\section{Potential mechanisms}

Several mechanisms may be hypothesized to underlie the observed changes in psychophysical thresholds for electrical stimulation of the cochlea including: (1) changes in the number of excitable elements, (2) altered characteristics of excitable elements, (3) altered current paths to the excitable elements, (4) altered central auditory pathways, and (5) behavioral changes. While we cannot prove any of these hypotheses based on the data in hand, it may be possible to reduce the alternatives. It is evident from the differences in effects of stimulus waveform that there must be at least two mechanisms, one that affects thresholds for all signals equally (in $\mathrm{dB}$ ) and one that has the greatest influence on thresholds for long-phase-duration signals.

It is evident from the studies using fixed-duration pulses and single pulses that the differential change for low frequency sinusoidal signals is attributable in part to the long phase durations of these signals. The effect of frequency, when phase duration was held constant, was small and did not correlate with the effect of frequency on thresholds of the sinusoidal stimuli, but the changes for single pulses as a function of phase duration correlated well with the changes for sinusoids. The dependence of threshold changes on phase duration suggests that they may be related to changes in the integration of current over time in each phase of the signal. This integration should be related, at least in part, to the membrane properties of single neural elements.

Alterations in the properties of the stimulated elements, such as changes in the lengths of periph- 
eral processes, or demyelination or remyelination of fibers, could produce phase-duration-dependent changes in thresholds since these changes would alter the membrane capacitance of the neurons. Effects of the length of the unmyelinated peripheral processes have been modeled by Colombo and Parkins (1987).

The changes over time in thresholds for single pulses were smaller than those for sinusoids measured over the same time period. This difference may reflect a multiplicative effect due to the much longer sample time available for the sinusoids, which had durations of several hundred $\mathrm{ms}$, as compared to the single pulses, which were $20 \mathrm{~ms}$ or less in duration. However, the mechanisms underlying this effect are not known.

Thresholds for $2 \mathrm{~ms} /$ phase pulses presented at $150 \mathrm{~Hz}$ showed greater changes over time than thresholds for $250 \mathrm{~Hz}$ sinusoids (which have a 2 ms phase duration) in the same implant over the same time period. These differences may be due to differences in the waveforms. Specifically, in the sinusoidal waveform, the signal is continuous so there is no interpulse interval. Thresholds for continuous and nearly continuous signals with long phase durations are typically higher than those for pulses of equal phase duration but lower frequency (e.g. Fig. 7) indicating that the proximity of the pulses may play a role in determining threshold.

Previous studies comparing thresholds from different subjects have shown inverse correlations between threshold levels and number of spiral ganglion cells in the stimulated cochleas (Pfingst and Sutton, 1983, 1984; Pfingst et al., 1985). The threshold differences between subjects correlated with nerve loss in these studies were of two types: those that occurred primarily for thresholds to low-frequency sinusoids and those that were similar in $\mathrm{dB}$ for all frequencies tested (e.g. see Fig. 2A in Pfingst et al., 1985) again suggesting that more than one mechanism may be involved.

Two features of our experimental animal subjects that must be considered as possible contributors to the threshold changes are the local administration of neomycin to deafen the cochlea just prior to implantation (in most cases) and the small amount of electrical stimulation administered to the deafened ears. It is clear that the acute effects of the neomycin were not a necessary condition for the short-term changes in thresholds since these changes were also seen in cases of reimplantation where no additional neomycin was given as well as in four cases where neomycin was not used at all. It is possible, however, that longer term changes in threshold were a result of the neomycin deafening. There is evidence of ongoing long-term degeneration following local administration of neomycin, implantation and electrical stimulation of the cochlea in monkeys (Miller et al., 1980) and following administration of systemic neomycin in cats (Leake and Hradek, 1988). Implantation and/or electrical stimulation of the cochlea have been shown to interact with the effects of ototoxic agents to retard degeneration (Lousteau, 1987; Leake et al., 1990). However, the relationship between the amount of stimulation and the degree of this effect is not known.

We would expect changes in thresholds if the current paths from the electrodes to the neurons were altered. A likely cause of such alteration would be tissue changes along these pathways including, but not restricted to, nerve loss or growth, and bone growth. If tissue grew on or very near the electrodes then we might expect associated changes in electrode impedance, but if the changes were more remote from the electrodes, impedance might be unaffected. Thus the poor correlation of impedance changes with the threshold changes reported in this paper only allows us to discount physical changes close to the electrodes. It is unlikely that tissue growth in the current paths from the electrodes to the excitable elements would cause a frequency or phase-duration-dependent attenuation of the signal, since the tissues of the cochlea are resistive in nature (Spelman et al., 1982, 1987). However, if alterations in the current paths were to result in changes in the site of excitation, for example from peripheral processes to cell bodies or central processes of auditory nerve fibers, then phase-duration-dependent effects would be plausible (see Loeb et al., 1983; van den Honert and Stypulkowski, 1984; Finley et al., 1989).

One of the possible causes of a change in current paths or distance from the electrodes to the excitable elements could be migration of the implant. Such migration has been demonstrated by successive radiographs in a human subject with 
a banded electrode array (Niparko et al., 1989). Unfortunately the electrode arrays used in the relevant cases in our study were too small to be seen by these methods.

Some restrictions can be placed on the potential mechanisms underlying the short-term changes in thresholds following implantation. It is unlikely that the decreases in thresholds commonly observed during the first few months following implantation were due to the subject's learning to hear the electrical stimulus. This is evidenced by five observations: (1) in many cases where testing began within the first few days after implantation, thresholds were as low as those seen after final stabilization; in these cases thresholds increased during the first few days after surgery and then decreased over a period of several weeks; (2) thresholds (at $1 \mathrm{kHz}$ ) in two animals not tested until about 2 months after implantation were stable from the first day of testing; (3) thresholds of animals experienced with electrical stimulation decreased as a function of time following reimplantation; (4) threshold changes were sometimes phase-duration-dependent; and (5) threshold changes were often greater than the entire dynamic range of hearing (from threshold to very loud sensations) measured before or after the change.

It seems unlikely that the short-term decreases in threshold following cochlear implantation were a result of increases in neural elements as the threshold changes were too rapid. In any case, neural sprouting in the implanted cochlea has, to our knowledge, not been observed.

By process of elimination, it would seem that the short-term changes in threshold following implantation were due either to changes in conductivity between the electrodes and the neural elements, or changes in the sensitivity of the neural elements themselves. The reversibility of the process, i.e. threshold increases followed by decreases, suggests either blocking and then clearing of current pathways or reversible damage to the neural elements. Histological studies in rats have shown that reversible swelling and other alterations in the peripheral processes of type I auditory nerve fibers can occur as a result of simply opening the cochlea and draining out some of the perilymph (Juiz et al., 1988; Kelly and Khanna, 1984; Rueda et al,,
1989). While the trauma of insertion of a scalar implant is certainly greater than that produccd by simply opening the cochlea, the reversible functional changes that we saw in monkeys bear an intriguing resemblance to the course of the histological changes seen in the studies in rats.

The long-term alterations in thresholds were typically increases from a previously stable baseline or from a relative low threshold after the short-term decrease. It is unlikely that these were due to behavioral changes since thresholds for acoustic stimuli to the nonimplanted ear remained stable during the time when thresholds for the electrical stimuli were changing. Also, as mentioned above, the changes in electrical thresholds were often larger than the entire dynamic range of hearing measured before the changes occurred, and in some cases they were stimulus-dependent. It is difficult to distinguish among the remaining alternative mechanisms: nerve loss, alteration in neural response characteristics, changes in current pathways, or central-pathway changes. It seems reasonable, however that those changes that were specific to longer-phase-duration signals were more likely to involve changes in the neural elements excited, the site of excitation, or central processing, while those that affect thresholds for all phase durations were more likely to involve alterations of the current levels at the excitable elements but not a change in the site on the elements where spikes were initiated.

\section{Implications for other cases}

While the functional changes reported in this paper do not necessarily imply occurrence in other cases, there are certainly good reasons to look for such occurrences. The potential of short-term changes is particularly relevant to acute animal preparations that are used for electrophysiological studies. Typically these studies are conducted shortly after implantation and/or deafening of the ear. If thresholds are fluctuating or are abnormally high during this period, there is a danger of recording from a preparation that differs significantly from that in which most behavioral studies are conducted (Pfingst, 1988). Since short-term changes have been seen in both humans and monkeys following implantation, there is reason to 
suspect that they may occur in the subjects that are used in acute physiological experiments as well.

Changes in thresholds in experimental preparations may be useful for elucidating the relationship between functional characteristics of electrical stimulation and what elements are excited by electrically stimulating the cochlea, particularly where within-subject designs are desirable.

The short-term changes may be less noticeable in the clinical application of cochlear implants, since a subject's implant may not be activated until several weeks after surgery. However, threshold stability should be checked before processor adjustments are made, particularly since threshold decreases have been observed for up to 6 months following implants in some monkeys. More important may be changes that can occur months or years after the implant surgery. Correcting for changes in threshold may require frequency-specific adjustments of the processor, not just an overall gain adjustment.

It may be questioned whether changes that occur only in thresholds for long-phase-duration signals are relevant to subjects whose processors use only short-phase-duration signals. In terms of processor calibration, probably they are not. However, the possibility must be considered that the changes in long-phase-duration signals are indicative of pathological conditions that may lead to longer term changes in the responses to all signals. Although the mechanisms underlying these changes are not clear, it does seem likely that the changes are caused by physical changes in the cochlea and/or the excitable neural tissues. Thus they are of concern with regard to the long-term prospects for the implant.

\section{Acknowledgements}

This work was supported by NIH-NIDCD Grant DC00274. The helpful comments of Sandy Spelman on previous drafts of this manuscript are most appreciated. I also express appreciation to Patti Otto and Daniel Rai for technical assistance.

\section{References}

Colombo, J. and Parkins, C.W. (1987) A model of electrical excitation of the mammalian auditory nerve neuron. Hear. Res. 31, 287--312.
Eddington, D.K., Dobelle, W.H., Brackmann, D.E., Mladejovsky. M.G. and Parkin, J.L. (1978) Auditory prosthesis research with multiple channel intracochlear stimulation in man. Ann. Otol. Rhinol. Laryngol. (St. Louis) 87, Supplement 53, 1-39.

Finley, C.C., Wilson, B.S. and White, M.W. (1989) Models of neural responsiveness to electrical stimulation. In: J.M. Miller and F.A. Spelman (Eds.), Cochlear Implants. Models of the Electrically Stimulated Ear, Springer-Verlag, New York, pp. 55-93.

Juiz, J., Rueda, J. and Merchan, J.A. (1988) Reversible damage to the nerve fibres in the organ of Corti after surgical opening of the cochlea in the rat. Acta Otolaryngol. (Stockholm) 106, 29-33.

Kelly, J.P. and Khanna, S.M. (1984) Distribution of cochlear damage caused by the removal of the round window membrane. Hear. Res. 16, 109-126.

Leake, P.A. and Hradek, G.T. (1988) Cochlear pathology of long term neomycin induced deafness in cats. Hear. Res. 33, 11-34.

Leake, P.A., Hultcrantz, M., Snyder, R.L. Hradek, G.T. and Rebscher, S.J. (1990) Effects of chronic electrical stimulation in neonatally deafened cats. Abst. Assoc. Res. Otolaryngol. p. 328.

Loeb, G.E., White, M.W. and Jenkins, W.M. (1983) Biophysical considerations in electrical stimulation of the auditory nervous system. Ann. N.Y. Acad. Sci. 405, 123-136.

Lousteau, R.J. (1987) Increased spiral ganglion cell survival in electrically stimulated, deafened guinea pig cochleac. Laryngoscope 97, 836-842.

Michelson, R.P. (1971) Electrical stimulation of the human cochlea: A preliminary report. Arch. Otolaryngol. 93, 317323.

Miller, J.M., Sutton, D. and Webster, D.B. (1980) Brainstem histopathology following chronic scala tympani implantation in monkeys. Ann. Otol. Rhinol. Laryngol. (St. Louis) 89, Supplement $66,15-17$.

Niparko, J.K., Zappia, J.J., Altschuler, R.A., Miller, J.M. Oviatt, D.L. and Kemink, J.L. (1989) Evaluation of the temporal bones of a human cochlear implant patient. Am. Neurotology Soc. Prog. Abst. 24, 33-34.

Pfingst, B.E. (1988) Comparisons of psychophysical and neurophysiological studies of cochlear implants. Hear. Res. 34, 243-252.

Pfingst, B.E. (1989a) Changes over time in thresholds for electrical stimulation of the cochlea. Abst. Assoc. Res. Otolaryngol. pp. $270-271$.

Pfingst, B.E. (1989b) Psychophysical constraints on biophysi$\mathrm{cal} /$ neural models of threshold. In: J.M. Miller and F.A. Spelman (Eds.), Cochlear Implants. Models of the Electrically Stimulated Cochlea, Springer-Verlag, New York, pp. 161-183.

Pfingst, B.E. and Sutton, D. (1983) Relation of cochlear implant function to histopathology in monkeys. Ann. N.Y. Acad. Sci. 405, 224-239.

Pfingst, B.E. and Sutton, D. (1984) Relation of psychophysical thresholds for electrical stimuli to auditory nerve survival: Summary of results from 18 scala tympani implants. Abst. Seventh Midwinter Res. Meeting. Assoc. Res. Otolaryngol., p. 10 
Pfingst, B.E., Hienz, R., Kimm, J. and Miller, J.M. (1975) Reaction-time procedure for measurement of hearing. I. Suprathreshold functions. J. Acoust. Soc. Am. 57, 421-430.

Pfingst, B.E., Donaldson, J.A., Miller, J.M. and Spelman, F.A. (1979) Psychophysical evaluation of cochlear prostheses in a monkey model. Ann. Otol. Rhinol. Laryngol. (St. Louis) $88,613-625$.

Pfingst, B.E., Burnett, P.A. and Sutton, D. (1983) Intensity discrimination with cochlear implants. J. Acoust. Soc. Am. $73,1283-1292$.

Pfingst, B.E., Glass, I., Spelman, F.A. and Sutton, D. (1985) Psychophysical studies of cochlear implants in monkeys: Clinical implications. In: R.A. Schindler and M.M. Merzenich (Eds.), Cochlear Implants, Raven Press, New York, pp. 305-321.

Pfingst, B.E., Albrektsson, T., Tjellström, A., Miller, J.M., Zappia, J., Xue, X. and Weiser, F. (1989) Chronic skull anchored percutaneous implants in nonhuman primates. $J$. Neurosci. Methods 29, 207-216.

Rueda, J., Juiz, J.M. and Merchan, J.A. (1989) Surgical trauma to the cochlea results in reversible damage to spiral gan- glion type I neurons. Acta Otolaryngol. (Stockholm) 107, $59-62$.

Shannon, R.V. (1989) A model of threshold for pulsatile electrical stimulation of cochlear implants. Hear. Res. 40. 197. 204.

Spelman, F.A., Pfingst, B.E. and Miller, J.M. (1978) A constant-current stimulator for use with chronic cochlear implants. Proc: San Diego Biomed. Symp. 17, 1-3.

Spelman, F.A., Clopton, B.M. and Pfingst, B.E. (1982) Tissue impedance and current flow in the implanted ear: Implications for the cochlear prosthesis. Ann. Otol. Rhinol. Laryngol. (St. Louis) 91, Supplement 98, 3-8.

Spelman, F.A., Clopton, B.M. and Suesserman, M.F. (1987) Measurements of the resistivity of bony tissues of the cochlea. Proceedings of the IEEE/EMBS 4, 191-192.

Van den Honert, C. and Stypulkowski, P.H. (1984) Physiological properties of the electrically stimulated auditory nerve. II. Single fiber recordings. Hear. Res. 14, 225-243.

Xue, X. and Pfingst, B.E. (1989) Inner ear implants for experimental electrical stimulation of auditory nerve arrays. J. Neurosci. Methods 28, 189-196. 